\title{
FUCIDIN-RESISTANT STAPHYLOCOCCI IN CURRENT HOSPITAL PRACTICE
}

\author{
J. R. Pattison and P. E. Mansell \\ Department of Bacteriology, The School of Pathology, The Middlesex Hospital \\ Medical School, London WIP 7LD
}

THE initial reports of the use of fucidin demonstrated the ease with which Staphylococcus aureus became resistant in vitro, apparently because of the presence in any large population of a small number of fucidin-resistant mutants (Barber and Waterworth, 1962; Godtfredsen, Roholt and Tybring, 1962; Hilson, 1962; Jensen, 1962; Taylor and Bloor, 1962). The reported frequency with which resistance developed during the clinical use of fucidin was variable (Newman et al., 1962; Taylor and Bloor; Jensen and Kiær, 1964), but in one series it occurred in 46 per cent. of patients with burns treated with the antibiotic (Lowbury et al., 1962). These observations led to the recommendation that fucidin should be used in combination with another antibiotic in the treatment of staphylococcal infections (Hilson; Lowbury et al.; Jensen and Kiær). The emergence of resistance following such combined therapy has been reported only rarely (Jensen and Lassen, 1969; Wright and Harper, 1970; Rowling, 1970). We observed a relapse of staphylococcal septicaemia (incident no. 6 in the present series) associated with the development of resistance to fucidin in a patient receiving combined therapy with cloxacillin and fucidin, and this prompted a review of fucidin resistance among staphylococci isolated in general hospital practice.

\section{MATERIALS AND METHODS}

\section{Organisms}

All the cultures of $S$. aureus referred to were isolated in the course of routine bacteriological examinations of clinical specimens that had been submitted for examination at the discretion of the clinicians, the majority during the investigation of frank or occult sepsis and the remainder as part of the screening routine of the various departments. Approximately 90 per cent. of specimens were obtained from in-patients and staff of the Middlesex Hospital Group and the remainder from patients attending the Casualty Department and from general practitioners in the area.

All organisms were tube-coagulase positive. Phage typing with the standard set of phages was performed in the Department.

\section{Antibiotic sensitivity}

Disk-testing. Resistant strains were first identified by disk-testing pooled samples of several colonies from the primary cultures. Tests were performed at $30^{\circ} \mathrm{C}$ on 5 per cent. lysed horse-blood agar plates with Multodiscs (Oxoid) containing penicillin 1.5 units, methicillin $10 \mu \mathrm{g}$, erythromycin $10 \mu \mathrm{g}$, tetracycline $10 \mu \mathrm{g}$, sodium fusidate $10 \mu \mathrm{g}$ and cotri-

Received 24 Oct. 1972; accepted 26 Nov. 1972.

J. MED. MICROBIOL.-WOL. 6 (1973) 
moxazole (23.5 $\mu \mathrm{g}$ sulphamethoxazole, $1.25 \mu \mathrm{g}$ trimethoprim). Tests of the Oxford staphylococcus (NCTC no. 6571) were performed concurrently.

Minimal inhibitory concentration (MIC). The MIC of fucidin (sodium fusidate, Leo Laboratories, Hayes, Middx.) was determined by a doubling-dilution technique in nutrient broth, $p \mathrm{H} \mathrm{7.0.}$. The inoculum was $0.03 \mathrm{ml}$ of a diluted overnight broth culture of the organism (c. $10^{3}$ viable organisms). All cultures were incubated at $37^{\circ} \mathrm{C}$ for $16-18 \mathrm{hr}$ and the MIC was taken as the lowest concentration in which growth was not visible.

\section{Bacterial multiplication}

This was assessed, after appropriate dilution of cultures in 1/4 strength Ringer's solution, by surface-colony counts per drop of diluted culture on plates of Columbia Agar (Oxoid CM331) enriched with 5 per cent. defibrinated horse blood (Oxoid SR50).

Generation times were calculated according to the method of Bulow-Sørensen (1962) from growth curves derived from results obtained by means of the above technique. Initial viable counts were $c .10^{3}$ organisms per $\mathrm{ml}$. Experiments indicated that the reproducibility of results was \pm 8 per cent.

\section{Resistance transfer}

Six-hr broth cultures of the fucidin-resistant, tetracycline-sensitive donor strains and the fucidin-sensitive, tetracycline-resistant recipient strains were mixed in fresh nutrient broth at a ratio of one donor cell to 100 recipient cells. The mixtures were incubated at $37^{\circ} \mathrm{C}$ for $18 \mathrm{hr}$ and suitable dilutions were dropped on to nutrient agar plates containing fucidin, $2 \mu \mathrm{g}$ per ml, and tetracycline, $10 \mu \mathrm{g}$ per ml. Plates were incubated for $48 \mathrm{hr}$ at $37^{\circ} \mathrm{C}$.

\section{RESULTS}

Fucidin-resistant $S$. aureus was detected during the routine bacteriological examination of specimens from 17 patients in 1971 . These represent 1.4 per cent. of the total isolates of $S$. aureus from patients during that time, counting only one isolate of the same strain from each patient. Because the isolates of fucidin-resistant $S$. aureus from each of two pairs of the patients are clearly of the same strain, only 15 test organisms (strains 1-15) have been investigated in the in-vitro studies.

\section{Case reports}

Incident 1.-S. aureus strain 1, not typable with the standard set of phages, was isolated from the sputum of a patient with fatal bronchopneumonia. The patient had had no fucidin or other antibiotics in the previous $12 \mathrm{mth}$ but was receiving ampicillin at the time of the isolation.

Incident 2.-S. aureus strain 2 , phage-typing pattern $54 / 84 / 85$, was isolated from the pustular lesions in the groin of a 9-day-old baby. It was not known whether the mother was a carrier of this strain at the time, but she had not been treated with fucidin or any other antibiotic in the previous $12 \mathrm{mth}$.

Incident 3.-S. aureus strain 3, phage-typing pattern $52 / 52 \mathrm{~A} / 80 / 47 / 54 / 75 / 84$, was isolated from the infected eczematous lesions of two patients.

(a) Patient D. J. was admitted in Apr. 1971, when strain 3 was isolated from skin and nose. The patient had had no fucidin or any other specific antistaphylococcal treatment in the past $2 \mathrm{yr}$ but had been treated with systemic and topical steroids for the previous $12 \mathrm{mth}$.

(b) Patient F. D. had been admitted to the same ward in Mar. 1971, when a fucidinsensitive $S$. aureus, phage-typing pattern 79 , was isolated from the skin and nose. The patient was treated with fucidin ointment from 29 Mar. 1971 to $10 \mathrm{Apr}$. 1971, and on the 11 Apr. 1971 fucidin-sensitive $S$. aureus, phage-typing pattern 79 , was isolated from the nasal swab whilst skin swabs yielded strain 3 . This staphylococcus disappeared from the 
skin lesions after the application of chlortetracycline cream and the patient was discharged on 3 May 1971.

Incident 4.-S. aureus strain 4, phage-typing pattern 53/84, was isolated from pus associated with ingrowing toe-nails. The patient had not had fucidin or any other antibiotic in the previous 2 yr.

Incident 5.-S. aureus strain 5, phage-typing pattern 53/54/75/77, was isolated from the throat swab of a nurse complaining of a sore throat. She had never been treated with fucidin nor been concerned with its administration but had had a course of penicillin $3 \mathrm{mth}$ previously. Six mth later $S$. aureus could not be isolated from nose and throat swabs.

Incident 6.-S. aureus strain 6, not typable with the standard set of phages, was isolated from the blood of a patient with staphylococcal septicaemia secondary to an intramuscular abscess.

Initially, wound swabs and blood cultures had yielded a fucidin-sensitive $S$. aureus which was also untypable with the standard phages. The patient was treated with cloxacillin (1 $\mathrm{g}$ intramuscularly four times daily for 4 days, $500 \mathrm{mg}$ intramuscularly four times daily for 4 days, and $250 \mathrm{mg}$ orally four times daily for 9 days) and fucidin ( $500 \mathrm{mg}$ orally four times daily for 4 days and $250 \mathrm{mg}$ orally four times daily for 12 days), and became clinically well and apyrexial after 14 days' treatment.

However, 12 days after the withdrawal of antibiotic treatment, the patient suffered a relapse of septicaemia although the abscess had drained and remained clinically quiescent. Blood cultures yielded strain 6 , which was resistant to fucidin but sensitive to cloxacillin; subsequent treatment with the latter antibiotic led to complete recovery. The strains isolated from this patient, though untypable with the standard set of phages, were all lysed by the experimental phage WH1 ("international" no. 93; Dr M. T. Parker, Staphylococcus Reference Laboratory, Central Public Health Laboratory, Colindale Avenue, London NW9 5HT).

Incident 7.-S. aureus strain 7, phage-typing pattern 53, was isolated from the nasal swab of a patient admitted for reduction of a fractured nose in Aug. 1971. The patient had applied fucidin ointment to facial impetigo lesions in Oct. 1969.

Incident 8.-S. aureus strain 8 , phage-typing pattern $79 / 54$, was isolated repeatedly from the skin lesions of a patient with reticulosis of the skin who had had several courses of cloxacillin for $S$. aureus infection at another hospital during the previous $18 \mathrm{mth}$. The staphylococci isolated were not tested for fucidin sensitivity or phage typed. The patient had not had fucidin at any time but had had, in addition to the cloxacillin, repeated courses of systemic and topical steroids and one course of ampicillin during the previous 2 yr.

Incident 9.-S. aureus strain 9, not typable with the standard set of phages, was isolated from the septic spots of a patient who had been applying fucidin ointment for some weeks before the isolation of the strain.

Incident 10. $-S$. aureus strain 10 , phage-typing pattern $79 / 42 \mathrm{E} / 54$, was isolated repeatedly from the sputum of a patient with lung metastases secondary to carcinoma of the cervix. The patient had not been treated with fucidin but had had one course of ampicillin and tetracycline and two of cotrimoxazole in the past $12 \mathrm{mth}$. At the time of the isolations the patient was receiving systemic steroids and intermittent radiotherapy and cyclophosphamide.

Incident 11.-S. aureus strain 11, phage-typing pattern $77 /+$, was isolated from the nose and throat swab of a 3-yr-old child admitted for neurological investigations. The patient had never been treated with fucidin but had had courses of penicillin and tetracycline in the past year.

Incident $12 .-S$. aureus strain 12 , phage-typing pattern $53 /+$, was isolated from the sputum of a patient with carcinoma of the bronchus who was admitted for radiotherapy. The patient had not had fucidin in the past 2 yr but a productive cough was being treated with tetracycline at the time of isolation of the test strain.

Incident 13.-S. aureus strain 13, phage-typing pattern $29 / 42 \mathrm{E} / 81$, was isolated from the nose swab of a 9-day-old baby (strain 13a). The mother was a nasal carrier of the 
same strain and had applied fucidin ointment for a staphylococcal skin infection $12 \mathrm{mth}$ previously (strain 13b).

Incident 14.-S. aureus strain 14, phage-typing pattern 81 , was isolated from the wound swab of a patient who had undergone repair of a hernia in Aug. 1971. In Sept. 1971 a fucidin- sensitive $S$. aureus, phage-typing pattern $29 / 88$, was isolated from the infected wound, and a 12-day course of oral fucidin ( $250 \mathrm{mg}$ four times daily) was started. In Dec. 1971 $S$. aureus strain 14 was isolated from the wound, which contained nylon suture material and a small collection of pus.

Incident 15.-S. aureus strain 15, not typable with the standard set of phages, was isolated from the wound swab of a patient with a cut hand. The patient had not received fucidin or any other antimicrobial treatment in the past $2 \mathrm{yr}$.

\section{In-vitro studies}

Antibiotic sensitivity. All the fucidin-resistant strains isolated in 1971 had the same pattern of sensitivity to the antibiotics tested. On disk-testing they were sensitive to tetracycline, erythromycin, methicillin, and cotrimoxazole but resistant to penicillin and fucidin. Fourteen strains (nos. 2-15) were kept and were available for further tests.

There is evidence (Evans and Waterworth, 1966) that in certain strains of $S$. aureus resistance to penicillin and fucidin are linked on a single, unstable plasmid. However, the fucidin resistance of 14 strains (nos. 2-15) has been stable both on storage for 12-18 mth and on repeated subculture in the absence of fucidin (see below). Moreover, the fucidin-sensitive parent strain of the fucidin-resistant strain 6 was resistant to penicillin. Loss of penicillin resistance has not yet been observed during the in-vitro testing of the fucidin-resistant strains.

Effect of fucidin. The MIC of fucidin for the strains available for testing was initially found to be either 12.5 or $25.0 \mu \mathrm{g}$ per ml, except for strain 6 , for which the MIC was $6.25 \mu \mathrm{g}$ per ml, and strain 8 , for which it was $100 \mu \mathrm{g}$ per ml (table I). This resistance proved to be largely stable, in that 50 passages in fucidin-free nutrient broth resulted in a fall in MIC of at most 1 or 2 doubling dilutions (table I).

The serum levels of fucidin in patients being treated with the manufacturer's recommended dose will often be well in excess of the MIC for these resistant strains, suggesting that standard treatment might be able to eradicate them. However, the potential resistance of these strains is only partially expressed. Serial subculture even in low concentrations of fucidin raised the MIC for all strains except one at least four-fold (table I). Moreover, subculture of selected strains in increasing concentrations of fucidin raised the MIC to $400 \mu \mathrm{g}$ per ml; and subsequently it is not until at least 50 subcultures in the absence of fucidin that the MIC began to approach its original lower level (table I).

Fucidin resistance by disk-testing of 30 to 50 individual colonies from all the primary cultures available (nos. 7-15) indicated that these strains consisted entirely of resistant variants. Moreover, when the MIC of these strains decreased on subculture in fucidin-free medium (table I), disk testing of individual colonies indicated that this reduction was due to a uniform lowering of resistance rather than to the appearance of a population of fully sensitive 
variants. Although not all strains were available as the primary culture of the clinical material, the in-vitro properties of those that were taken from the storage nutrient agar slopes did not differ substantially from those available in primary culture (table I).

In only one case (no. 6) was a fully sensitive (MIC $0.4 \mu \mathrm{g}$ per ml fucidin) $S$. aureus of the same phage-typing pattern isolated before the appearance of the resistant strain. Examination of all the primary cultures of the sensitive

TABLE I

In-vitro properties of the fucidin-resistant staphylococci

\begin{tabular}{|c|c|c|c|c|c|c|}
\hline \multirow[b]{2}{*}{$\begin{array}{l}\text { Strain } \\
\text { number* }\end{array}$} & \multirow[b]{2}{*}{$\begin{array}{l}\text { Source of } \\
\text { test sample }\end{array}$} & \multicolumn{5}{|c|}{ Minimum inhibitory concentrations of fucidin for the stated strain } \\
\hline & & Original & $\begin{array}{l}\text { (2) } \\
\text { (1) after } 50 \\
\text { subcultures } \\
\text { in absence } \\
\text { of fucidin }\end{array}$ & $\begin{array}{l}\text { (3) } \\
\text { (2) followed by } \\
10 \text { subcultures } \\
\text { in } 5 \mu \mathrm{g} \text { per } \mathrm{ml} \\
\text { fucidin }\end{array}$ & $\begin{array}{l}\text { (4) } \\
\text { (3) followed by } \\
12 \text { subcultures } \\
\text { in increasing } \\
\text { concentration } \\
\text { of fucidin }\end{array}$ & $\begin{array}{l}\text { (4) followed by } \\
50 \text { subcultures } \\
\text { in absence of } \\
\text { fucidin }\end{array}$ \\
\hline 2 & Storage slope & $12 \cdot 5$ & $6 \cdot 25$ & 50 & & \\
\hline 3 & Storage slope & 12.5 & 6.25 & 50 & 400 & $\ddot{25}$ \\
\hline 4 & Storage slope & 12.5 & 6.25 & 25 & & \\
\hline 5 & Storage slope & 12.5 & $6 \cdot 25$ & 50 & 400 & 25 \\
\hline 6 & Primary culture & $6 \cdot 25$ & $3 \cdot 12$ & 25 & 400 & 12.5 \\
\hline 7 & Primary culture & 12.5 & 6.25 & 50 & & \\
\hline 8 & Primary culture & 100 & 25 & 50 & 400 & 100 \\
\hline 9 & Primary culture & 25 & 6.25 & 50 & $\ldots$ & $\ldots$ \\
\hline 10 & Primary culture & $12 \cdot 5$ & $6 \cdot 25$ & 50 & $\ldots$ & $\ldots$ \\
\hline 11 & Primary culture & 12.5 & $12 \cdot 5$ & $12 \cdot 5$ & $\ldots$ & $\ldots$ \\
\hline 12 & Primary culture & 25 & $6 \cdot 25$ & 25 & $\ldots$ & .... \\
\hline 13 & Primary culture & 25 & 6.25 & 25 & $\ldots$ & $\ldots$ \\
\hline 14 & Primary culture & $12 \cdot 5$ & $6 \cdot 25$ & 25 & $\ldots$ & $\ldots$ \\
\hline 15 & Primary culture & 25 & $6 \cdot 25$ & 25 & $\ldots$ & $\ldots$ \\
\hline
\end{tabular}

$\ldots=$ Not tested.

* Strain no. 1 was not available for testing.

variant indicated the presence of a minor population of resistant variants at a rate of one in $10^{5}-10^{6}$ sensitive cells.

All the fucidin-resistant strains that had been isolated from clinical specimens had generation times of 23-28 min. compared with a generation time of $24 \mathrm{~min}$. for the Oxford staphylococcus (table II). In particular, the naturally occurring fucidin-resistant variant of strain 6 had exactly the same generation time as the fully sensitive variant of the same phage-typing pattern; but when fucidin-resistant variants were freshly selected in vitro from large inocula of the fucidin-sensitive parent strain the generation time was $45 \mathrm{~min}$. (table II). Subsequently the generation time of these freshly isolated resistant variants fell to 24 min. within six serial subcultures.

Experiments with three randomly selected fucidin-sensitive staphylococci showed that the fucidin-resistant variants, present as a minor population in all three strains, had generation times initially of 48-60 min. (table II), and after six subcultures this was not significantly different for any of the three strains. 
Effect of prednisolone. Only six of the 15 test strains were isolated from patients who had previously been treated with fucidin. Of the remaining nine, three were isolated from patients who had received steroid therapy. Because fucidin also has a steroid-like structure it was considered possible that treatment with steroids might tend to select for fucidin-resistant staphylococci. Accordingly, six fucidin-sensitive staphylococci were selected at random from those isolated in the routine laboratory and a large inoculum of each of these was shown to include a minority (on average 1 in 105) of

TABLE II

Comparison of the generation times of fucidin-resistant and -sensitive staphylococci

\begin{tabular}{|c|c|c|c|c|c|c|c|c|}
\hline \multicolumn{9}{|c|}{ Generation time (min.) of } \\
\hline \multirow{4}{*}{$\begin{array}{l}\text { the Oxford } \\
\text { staphylococcus } \\
\text { (NCTC6571) }\end{array}$} & \multicolumn{5}{|c|}{ strains isolated from clinical specimens } & \multirow{3}{*}{\multicolumn{3}{|c|}{$\begin{array}{l}\text { fucidin-resistant } \\
\text { variants selected in } \\
\text { vitro from random } \\
\text { strains }\end{array}$}} \\
\hline & \multirow{3}{*}{$\begin{array}{l}\text { nos. 2-5 } \\
\text { fucidin } \\
\text { resistant }\end{array}$} & \multicolumn{3}{|c|}{ no. 6} & \multirow{3}{*}{$\begin{array}{l}\text { nos. } 7-15 \\
\text { fucidin } \\
\text { resistant }\end{array}$} & & & \\
\hline & & \multirow{2}{*}{$\begin{array}{l}\text { fucidin- } \\
\text { sensitive } \\
\text { variant }\end{array}$} & \multirow{2}{*}{\multicolumn{2}{|c|}{$\begin{array}{c}\text { fucidin- } \\
\text { resistant } \\
\text { variant }\end{array}$}} & & & & \\
\hline & & & & & & A & B & $\overrightarrow{\mathrm{C}}$ \\
\hline 24 & $24-27^{*}$ & $24^{*}$ & $24^{*}$ & $45 \dagger$ & $23-28 *$ & $48 \ddagger$ & $58 \ddagger$ & $60 \ddagger$ \\
\hline
\end{tabular}

* Isolated from clinical specimens.

† Selected in vitro from sensitive parent strain; generation time fell to $24 \mathrm{~min}$. after six further subcultures.

$\$$ Selected in vitro from sensitive parent strain; generation time not significantly different after six further subcultures.

fucidin-resistant variants. The frequency of these variants was compared in cultures grown in the absence of prednisolone (prednisolone sodium phosphate BP; Merck, Sharp \& Dohme Ltd, Hoddesdon, Herts.) and in the presence of $(a) 5,(b) 50$, and (c) $4000 \mu \mathrm{g}$ per $\mathrm{ml}$ prednisolone, which were respectively concentrations that $(a)$ have little effect, $(b)$ maximally stimulate and $(c)$ inhibit oxygen consumption by $S$. aureus (Raab, 1971). After 50 subcultures in such media, the frequency with which resistant variants were isolated was no different in any of the cultures, and was in the range 4-20 per million sensitive variants for all six strains under all four growth conditions. Moreover, the rate of growth of the fucidin-resistant strains was no different from that of the fucidin-sensitive strains in any of the concentrations of prednisolone.

Resistance transfer. The appearance of fucidin resistance in a variety of different strains in the hospital suggested that the determinant might be readily transferred between strains. All 14 fucidin-resistant strains (nos. 2-15) were used as prospective donors, and five strains of $S$. aureus resistant to tetracycline but sensitive to penicillin, methicillin, erythromycin, cotrimoxazole, and fucidin were used as prospective recipients. However, transfer of resistance could not 
be demonstrated in mixed culture from any of the fucidin-resistant strains to any of the fucidin-sensitive strains.

\section{Discussion}

The fucidin-resistant strains isolated represent 1.4 per cent. of staphylococci isolated in 1971. This is not substantially different from the figure for 1970 ( 1.1 per cent.) and for the first $6 \mathrm{mth}$ of 1972 (1.0 per cent.). The resistant strains described have undoubtedly retained their human pathogenicity. Over half the strains isolated (nos. 1, 2, 3, 4, 6, 8, 9, 14 and 15) were obviously associated with major or minor sepsis.

Six of the 15 strains were isolated from patients who had received fucidin therapy. One of these patients had been treated with a combination of fucidin and cloxacillin. Previous reports of the emergence of fucidin resistance during combined antibiotic therapy have indicated that it is associated with infection of large areas of necrotic, devitalised tissue (Jensen and Lassen, 1969; Rowling 1970 ) or with the difficult therapeutic problem of cystic fibrosis (Wright and Harper, 1970). The case reported here in which the primary infection was a large intramuscular abscess conforms to this pattern. All authors stress the importance of prolonged full-dosage therapy in such situations; in the case described here the recommended dose was halved after only 4 days' treatment and discontinued only 2 days after the patient became apyrexial.

One strain (no. 14) was isolated from a patient treated with oral fucidin alone, and again the low dosage and presence of pus and foreign body in the wound are notable. Four other strains (nos. 3, 7, 9 and 13b) were isolated from patients who had applied fucidin ointment.

Strain 6 undoubtedly appeared by a process of selection of a minority population of resistant variants from a largely sensitive population of staphylococci of the same phage-typing pattern. In two other instances (nos. $3 \mathrm{~b}$ and 14) fucidin-resistant strains colonised and infected sites from which sensitive staphylococci of a different phage-typing pattern had been eliminated by treatment with fucidin. In one (no. 3b) the acquisition was by a process of hospital cross-infection, but in the other infection was acquired while the patient was being treated as an out-patient.

No such information is available for the other three strains isolated from patients who had received fucidin therapy, but it is clear that the resistant strains once established can persist in the carriage state for 12-21 mth (nos. 7 and 13b). No other factor that might select for fucidin resistance could be identified in the nine patients who had not been treated with fucidin and from whom fucidin-resistant strains were isolated.

Except in the case of the patients from whom strains 2, 5 and 6 were isolated, it was clear that all patients were either admitted already colonised with the resistant strains (nos. 1, 3a, 4, 7, 8, 9, 10,11, 12 and 13b), were colonised only as outpatients (nos. 14 and 15), or acquired the resistant strains in hospital from patients admitted carrying the strain (nos. $3 b$ and 13a). It appears therefore that these strains represent the pool of stable wild-type fucidin-resistant strains. 
That there was not also a pool of fucidin-resistant strains amongst the hospital staphylococci was thought initially to be due to the restricted use of fucidin in the Hospital Group. It is the recommendation of the Department of Bacteriology that fucidin should always be used in combination and only for infections due to methicillin-resistant strains, or for serious sepsis where rapid response, good penetration, or both, are desirable (e.g., septicaemia, endocarditis, osteomyelitis). Methicillin-resistant strains do not occur frequently in the Middlesex Hospital (0.9 per cent. of all strains isolated in 1971) and in

TABLE III

Amount of fucidin purchased by the Middlesex Hospital

\begin{tabular}{|c|c|c|c|c|c|c|c|c|}
\hline \multirow[b]{3}{*}{ Unit } & \multicolumn{8}{|c|}{ Number of stated units of fucidin purchased in } \\
\hline & \multicolumn{4}{|c|}{1970} & \multicolumn{4}{|c|}{1971} \\
\hline & $\begin{array}{l}\text { 1st } \\
\text { quarter }\end{array}$ & $\begin{array}{l}\text { 2nd } \\
\text { quarter }\end{array}$ & $\begin{array}{l}\text { 3rd } \\
\text { quarter }\end{array}$ & $\begin{array}{l}\text { 4th } \\
\text { quarter }\end{array}$ & $\begin{array}{l}\text { 1st } \\
\text { quarter }\end{array}$ & $\begin{array}{l}\text { 2nd } \\
\text { quarter }\end{array}$ & $\begin{array}{l}\text { 3rd } \\
\text { quarter }\end{array}$ & $\begin{array}{l}\text { 4th } \\
\text { quarte }\end{array}$ \\
\hline $\begin{array}{l}\text { Capsules and tablets } \\
(250 \mathrm{mg})\end{array}$ & 750 & 1500 & 500 & 1000 & 2500 & 2250 & 3950 & 2850 \\
\hline $\begin{array}{l}\text { Intravenous solution } \\
\quad(500 \mathrm{mg})\end{array}$ & 0 & 0 & 0 & 63 & 173 & 30 & 30 & 0 \\
\hline $\begin{array}{l}\text { Oral suspension }(60 \mathrm{ml} \text {; } \\
125 \mathrm{mg} \text { per } \mathrm{ml})\end{array}$ & $\mathbf{0}$ & 0 & 0 & 21 & 12 & $\mathbf{0}$ & 6 & 12 \\
\hline $\begin{array}{l}\text { Tins of tulle (ten pieces } \\
\text { per tin) }\end{array}$ & 36 & 24 & 24 & 0 & 30 & 24 & 24 & 30 \\
\hline Ointment (10 g) & 78 & 72 & 72 & 42 & 36 & 40 & 80 & 12 \\
\hline
\end{tabular}

1971 only two cases of staphylococcal septicaemia, one of staphylococcal endocarditis and not more than six cases of osteomyelitis were seen. However, the amount of fucidin purchased by the Middlesex Hospital Pharmacy (table III) indicates that the use of fucidin cannot have been restricted to the above indications. Unfortunately it was not possible in retrospect to trace exactly how and where this fucidin was used in the hospital group.

Fucidin has now been available for $10 \mathrm{yr}$ and the early impression (Taylor and Bloor, 1962; Crosbie, 1963) must remain that the appearance of fucidin resistance in clinical practice is much less common than would be anticipated from the ease with which staphylococci can become resistant in vitro.

This may be related to variations in the generation times of fucidin-sensitive and -resistant staphylococci; previous work (Bülow-Sørensen, 1962; Miller, 1971) has shown that the fucidin-resistant variants that exist as a minor population in large inocula of staphylococci have a prolonged generation time. We confirmed this for resistant variants freshly selected from large inocula of the sensitive parent of strain 6 . However, the resistant strain 6 that was isolated from clinical material had a generation time as fast as that of the 
parent strain, and subculture of freshly isolated resistant variants indicated that the generation time quickly decreases from $45 \mathrm{~min}$. to $24 \mathrm{~min}$., a property not shared by the resistant variants of three randomly selected fucidin-sensitive strains. It may be that only variants that have the capacity to accelerate their rate of multiplication can establish themselves as stable resistant clones.

Nevertheless, the potential for selecting stable, persistent, pathogenic strains of $S$. aureus that are resistant to fucidin clearly exists. The recommendation of the Bacteriology Department will therefore continue to be that fucidin is used only in combination with another antibiotic in those situations outlined earlier, and the occurrence of resistant strains in relation to treatment with fucidin will be monitored prospectively.

\section{SUMMARY}

Fifteen strains of $S$. aureus resistant to fucidin were isolated from 17 patients during the routine examination of clinical specimens in 1971 (1.4 per cent.). Over half of them were associated with sepsis, but only 40 per cent. were isolated from patients who had previously been treated with fucidin. In-vitro studies indicate that the strains have a stable basic level of resistance with the potential to increase this resistance to high levels on exposure to fucidin. The strains multiply as rapidly as fully sensitive strains, may be associated with persistent and relapsing sepsis and may persist in the carriage state for up to 21 mth.

We are grateful to Mr G. Bryan, Chief Pharmacist for supplying the information given in table III.

\section{REFERENCES}

Barber, Mary, and Waterworth, Pamela M. 1962. Antibacterial activity in vitro of fucidin. Lancet, $1,931$.

Bülow-Sørensen, P. 1962. Antibacterial effect of fucidin (ZN6') in vitro. Ugeskr. Laeg., 124, 745 (in Danish).

Crosbie, R. B. 1963. Treatment of staphylococcal infections with "fucidin". Brit. Med. $J ., 1,788$.

Evans, R. J., and Waterworth, Pamela M. 1966. Naturally-occurring fusidic acid resistance in staphylococci and its linkage to other resistances. J. Clin. Path., 19, 555.

Godtrredsen, W., Roholt, K., AND Tybring, L. 1962. Fucidin. A new orally active antibiotic. Lancet, 1, 928.

HiLsON, G. R. F. 1962. In-vitro studies of a new antibiotic (Fucidin). Lancet, 1, 932.

JENSEN, K. A. 1962. Bacteriological control of the effect of fucidin on straphylococcal infections. Ugeskr. Laeg., 124, 753 (in Danish).

JeNSEN, K. A., AND KIAR, INGER 1964. Fucidin. A study on problems of resistance. 1. Acta path. microbiol. scand., 60, 271.

JeNSEN, K. A., AND LASSEN, H. C. A. 1969. Combined treatment with antibacterial chemotherapeutical agents in staphylococcal infections. Quart.J. Med., N.S. 38, 91.

Lowbury, E. J. L., CASON, J. S., JACKSON, D. MaCG., and Miller, R. W. S. 1962. Fucidin for staphylococcal infection of burns. Lancet, 2, 478.

Miller, G. R. 1971. Fusidic-acid-resistant staphylococci. Antonie van Leeuwenhoek, 37, 313.

Newman, R. L., Bhat, K. M., Hackney, Ruth, Robinson, Christine, and Stewart, G. T. 1962. Fusidic acid: laboratory and clinical assessment. Brit. Med. J., 2, 1645. 
RAAB, W. P. 1971. The interaction of corticosteroids and antimicrobial agents used in topical therapy. Br. J. Derm., 84, 582.

RowLING, D. E. 1970. Further experience in the management of chronic osteomyelitis. $J$. Bone Jt Surg., 52B, 302.

TAYLOR, G., AND BLOOR, K. 1962. Antistaphylococcal activity of fucidin. Lancet, 1, 935. WrIGHT, G. L. T., AND HARPER, J. 1970. Fusidic acid and lincomycin therapy in staphylococcal infections in cystic fibrosis. Lancet, $1,9$. 